\title{
Search for Formation Criteria of Globular Cluster Systems
}

\author{
S. N. Nuritdinov ${ }^{1,2}$, K. T. Mirtadjieva ${ }^{1,2}$ and I. U. Tadjibaev ${ }^{1}$ \\ ${ }^{1}$ National University of Uzbekistan, Tashkent 700174, Uzbekistan \\ ${ }^{2}$ Ulugh Beg Astronomical Institute, Tashkent 700052, Uzbekistan
}

The research of early evolution stages and formation of galaxies requires creation of appropriate non-linear theory. The formation process of globular cluster systems (GCS) covers the long period of time as probably one begins from a dark matter state. So far it is difficult to simulate formation of GCS and galaxies ab initio. That's why it is necessary to construct exact analytically solvable models of non-linear non-stationary early stages of evolution of self-gravitating systems and revealing the instabilities on a background of non-equilibrium states. The formation and evolution of GCS at early stage of collapsing dark matter (or protogalaxy) can be explained by instability of the modes of the high oscillation degrees (Nuritdinov et al., 2000), which corresponds to rather small-scale perturbations of the density of collapsing system. The mode degree defines on the average the number of clusters. We study of the mode behavior of oscillations of high degrees, solving the non-stationary dispersion equation (NDE), for example,

$(1+\lambda \cos \psi) \frac{d^{2} \gamma_{\tau}}{d \psi^{2}}+\lambda \sin \psi \frac{d \gamma_{\tau}}{d \psi}+\gamma_{\tau}=(1+\lambda \cos \psi)^{3}(\lambda+\cos \psi)^{N-\tau} \cdot \sin ^{\tau-1} \psi A(\psi)$ at $N>>1$, where $\lambda=1-(2 T /|U|)_{0},(2 T /|U|)_{0}$ is the virial ratio at $\psi=0$ and $A(\psi)$ is unknown function of time.

Searching for an exact formation criteria of the GCS demands finding an exact asymptotic of the NDE. Using the asymptotic for the Legandre function $P_{N}(e)$ at $N>>1$ we found a numerical solution of the NDE.

We have concluded:

- GCS formation takes place in the background of collapsing dark matter only for very special conditions of $(2 T /|U|)_{0}<<1$.

- In spite of the fact that the modes of high degree are small- scale in comparison of the ellipsoidal mode $(2 ; 2)$, their maximal increments are close in many cases.

- At very large values of $N$ the instability increment is decreased.

- The analysis of special cases of the NDE shows that it is necessary to find an exact asymptotic in the case $N \rightarrow \infty$ taking into account the rotation effect. 